\title{
Early Diagnosis and Prevention of Repetitive Strain Injury Induced Carpal Tunnel Syndrome among Computer Users
}

\section{Sonia Agrawal ${ }^{1}$ and Rajajeyakumar $\mathbf{M}^{2^{*}}$}

${ }^{1}$ Jawaharlal Institute of Postgraduate Medical Education \& Research (JIPMER), Puducherry, India

${ }^{2}$ Department of Physiology, Trichy SRM Medical College Hospital \& Research Centre, Trichy, Tamil Nadu, India

\begin{abstract}
Carpal tunnel syndrome (CTS) is one of the most frequent compression neuropathies involving the median nerve during its course through the carpal tunnel. The prevalence in the general population is about 3.0-5.8 percent among women and $0.6-2.1 \%$ among men, based on both clinical symptoms and nerve conduction study (NCS). High stress and pressure in the carpal tunnel may lead to the development of CTS. This is now mostly accepted that exposure to hand-arm vibrations and combination of repetitive hand force use may be the causative factor. In the recent years, the widespread use of computers and laptops has lead to a matter of concern whether the use of these devices could be a risk factor in the causation of CTS. Hence this article reviews the relation between computer users and CTS, the methods of early diagnosis and ways to prevent it.
\end{abstract}

Keywords: Carpal Tunnel Syndrome; Disorders

\section{Aim}

To evaluate the methods for early diagnosis and the preventive strategies to reduce the occurrence of repetitive strain induced carpal tunnel syndrome in computer users [1-3].

\section{Introduction}

In this systemic mini review, we studied the association between the development of CTS and computer users and various methods for early diagnosis of CTS. The research for literature was carried out using online databases like Pub Med, Research Gate, MEDLINE and Google Scholar. The studies written in English and published in peer reviewed journals were considered. The original research paper data were included.

The keywords used were, carpal tunnel syndrome, computer users, median nerve, repetitive strain, musculo skeletal disorders, and computer professionals. The titles and abstracts for each study were thoroughly read. The references from each article were further searched upon to look for various other studies.

The studies were cross-sectional in nature. The studies had prospective design and included carpal tunnel syndrome as the outcome. Few studies also analyzed other risk factors associated with CTS. Studies to diagnose CTS were searched upon and included in the study. Also one study which evaluated the preventive measures for CTS was included. No cadaver studies were included and no scoring system was done to evaluate any of these studies.

\section{Results and Discussion}

In the literature search many studies about CTS were found but only few had data regarding association between CTS and computer users. 6 studies were reviewed for this study.

The cross-sectional study done by Sudha et al. [4], among office workers ( $n=470,55.6 \%$ - males) in Kuwait, reported the prevalence of self-administered CTS to be $18.7 \%$.The significant risk factors included obesity, female gender and number of co morbid conditions. However, the study could not show computer use to be a definitive risk factor for development of CTS. The reasons could be the self-administered nature of the questionnaire rather than clinical assessment per se.
A cross sectional study was conducted in India by Ali and Sathiyasekaran [5] found that, subjects $(n=4276)$ who used computers for more than 8 years and over 12 hours per day at a higher risk for CTS (OR 3.3, 4.9 and 2.5 respectively). There was no significant gender difference (men had twice the frequency of CTS compared to women), age or Body mass index. However, blinding was not described in the study. Also they found improper hand position to be associated with CTS though it was not statistically significant [5].

Another study conducted by Arslan et al. [6] in 50 patients with CTS, aimed to quantify the stiffness of median nerve by using acoustic radiation force impulse (ARFI) elastography. They showed that ARFI elastography was an efficient and recent technology in predicting the diseases and grading the severity among cases as compared to controls. This is a non-invasive technique and hence easy to administer. However the study included more females $(\mathrm{n}=48)$ as compared to males. They did not correlate the diagnosis of CTS in computer users [6].

Vahdatpour et al. [7], studied in 50 patients, the role of median nerve terminal latency index (mTLI) in diagnosis of CTS as compared to other electro diagnostic tests. They have reported that, the most sensitive neurophysiological evaluation in CTS to be mTLI (82\%) but the most specific was distal motor latency (98\%). After plotting the area under curve (AUC) in ROC, they found that fourth digit median-ulnar peak latency difference (PM4-U4), had better diagnostic reliability as compared to mTLI [7].

Azman D et al. [8] in their study assessed various ultrasonographic parameters in confirmation of CTS. The inlet cross-sectional area, inlet circumference and outlet cross-sectional area of the median nerve had increased UCs $(0.962,0.920$, and 0.913 , respectively), sensitivities

*Corresponding author: Rajajeyakumar M, Assistant Professor, Department of Physiology, Trichy SRM Medical College Hospital \& Research Centre, Trichy, Tamil Nadu, India, Tel: + 91 97513-82650; E-mail: rajakumar60@gmail.com

Received: March 15, 2018; Accepted: March 23, 2018; Published: March 30 2018

Citation: Agrawal S, Rajajeyakumar M (2018) Early Diagnosis and Prevention of Repetitive Strain Injury Induced Carpal Tunnel Syndrome among Computer Users. Clin Exp Psychol 4: 188. doi: 10.4172/2471-2701.1000188

Copyright: (c) 2018 Agrawal S, et al. This is an open-access article distributed under the terms of the Creative Commons Attribution License, which permits unrestricted use, distribution, and reproduction in any medium, provided the original author and source are credited. 
Citation: Agrawal S, Rajajeyakumar M (2018) Early Diagnosis and Prevention of Repetitive Strain Injury Induced Carpal Tunnel Syndrome among Computer Users. Clin Exp Psychol 4: 188. doi: 10.4172/2471-2701.1000188

Page 2 of 2

(87.4\%, 80.0\%, and 74.1\%), and specificities (94.6\%, 91.4\%, and 92.5\%) among single-measurement parameters [8].

Zidkova et al. [9], studied the role of preventive measure for CTS in automotive assembly workers. Introduction of preventive measures low the prevalence of median neuropathy from $18.3 \%$ in 2011 to $10.5 \%$ in 2013 ( $\mathrm{p}=0.003$ ). The preventive measures included, causes of CTS, changes of tools, switching of working tasks and thus decreasing repetitive wrist movements, transfer to less stress job or introducing more breaks in between work [9].

\section{Conclusion}

Very few studies have reported the association of CTS among computer or keyboard users. Various methods of diagnosing CTS have been studied but larger studies with more sample size are needed to establish the most efficient and cost effective test to diagnose CTS. As computer use for a long time can lead to repetitive strain induced CTS, the need for preventive measures is highly stressed upon. The authors recommend that long term studies with well-defined risk assessment and outcome measures are needed to formulate appropriate guidelines for preventive measures.

\section{References}

1. De Krom MC, Knipschild PG, Kester AD, Thijs CT, Boekkooi PF, et al. (1992) Carpal tunnel syndrome: prevalence in the general population. J Clin Epidemiol 45: 373-376.

2. Atroshi I, Gummesson C, Johnsson R, Ornstein E, Ranstam J, et al. (1999) Prevalence of carpal tunnel syndrome in a general population. JAMA 282: 153158.

3. Palmer KT, Harris EC, Coggon D (2007) Carpal tunnel syndrome and its relation to occupation: a systematic literature review. Occup Med (Lond) 57: 57-66.

4. Raman SR, Becher Al-Halabi, Hamdan E, Landry MD (2012) Prevalence and risk factors associated with self-reported carpal tunnel syndrome (CTS) among office workers in Kuwait. BMC Res Notes 5: 289.

5. Ali KM, Sathiyasekaran BW (2006) Computer professionals and Carpal Tunnel Syndrome (CTS). Int J Occup Saf Ergon 12: 319-325.

6. Arslan H, Yavuz A, İlgen F, Aycan A, Ozgokce M, et al. (2018) The efficiency of acoustic radiation force impulse (ARFI) elastography in the diagnosis and staging of carpal tunnel syndrome. Journal of Medical Ultrasonics.

7. Vahdatpour B, Khosrawi S, Chatraei M (2016) The role of median nerve terminal latency index in the diagnosis of carpal tunnel syndrome in comparison with other electrodiagnostic parameters. Adv Biomed Res 5: 110.

8. Azman D, Hrabac P, Demarin V (2017) Use of multiple ultrasonographic parameters in confirmation of carpal tunnel syndrome. J Ultrasound Med.

9. Žídková V, Nakládalová M, Zapletalová J, Nakládal Z, Kollárová H (2017) Experiences with preventing carpal tunnel syndrome in an automotive plant. Int J Occup Med Environ Health 30: 45-54. 